\title{
Terahertz polarizing component on cyclo-olefin polymer
}

\author{
A. Ferraro ${ }^{* 1,2}$, D. Zografopoulos, ${ }^{1}$ R. Caputo $^{2}$, and R. Beccherelli ${ }^{1}$ \\ ${ }^{I}$ Consiglio Nazionale delle Ricerche, Istituto per la Microelettronica e Microsistemi (CNR-IMM), Roma 00133, \\ Italy, \\ ${ }^{2}$ Department of Physics, University of Calabria, Via Ponte Bucci Cubo 33b, 87036 Rende, Italy.
}

Received February 07, 2017; accepted March 27, 2017; published March 31, 2017

\begin{abstract}
Wire-grid polarizers constitute a traditional component for the control of polarization in free-space devices that operate in a broad part of the electromagnetic spectrum. Here, we present an aluminiumbased $\mathrm{THz}$ wire grid polarizer, fabricated on a sub-wavelength thin flexible and conformal foil of Zeonor polymer having a thickness of $40 \mu \mathrm{m}$. The fabricated device, characterized by means of $\mathrm{THz}$ timedomain spectroscopy, exhibits a high extinction ratio between 30 and $45 \mathrm{~dB}$ in the $0.3-2.1 \mathrm{THz}$ range. The insertion losses oscillate between 0 and $1.1 \mathrm{~dB}$ and they stem almost exclusively from moderate Fabry-Perót reflections and it is engineered for vanishing at $2 \mathrm{THz}$ for operation with quantum cascade lasers.
\end{abstract}

The terahertz $(\mathrm{THz})$ frequency range is under intense investigation due to its applications in cross-disciplinary fields, such as secure short-range communications, lifescience, defence and security [1-8]. In this scenario, the development of new $\mathrm{THz}$ components able to manipulate the $\mathrm{THz}$ radiation is a primary issue. Several research groups have been working on the design and fabrication of such components, e.g. filters, beam splitters, phase shifters, and electromagnetic absorbers [9-17]. Analysing and controlling the polarization of waves is a key basic functionality and, by using different approaches, various $\mathrm{THz}$ polarizers have been so far developed [18-21]. In our previous work [22], we demonstrated an Al-based $\mathrm{THz}$ wire grid polarizer (WGP) on a cyclo-olefin polymer substrate with very low $\mathrm{THz}$ absorption losses and high mechanical flexibility, which was mainly optimised for controlling the polarization in the low $\mathrm{THz}$ range. Here, we extend our work and demonstrate operation at about $2.0 \mathrm{THz}$ by using a $40 \mu \mathrm{m}$ thick Zeonor foil. This targeted frequency falls in the range delivered by Quantum Cascade Lasers (QCL) [23]. The fabricated WGP possesses a high extinction ratio (ER) and extremely low insertion losses (IL). The latter are mainly influenced by the Fabry-Pérot effect but vanish at the targeted frequency, thus producing no undesired reflection towards the QCLs. In general, thanks to the very low $\mathrm{THz}$ absorption losses of the polymer and its good processability, it is possible to engineer the matching frequency by properly selecting the substrate thickness. The properties of the WGP depend on its pitch $p$, the width of the metallic stripes $w$ and the fill factor $(F)$,

\footnotetext{
*E-mail: antonio.ferraro@artov.imm.cnr.it
}

defined as the ratio between the stripe width and the pitch of the one-dimensional periodic lattice. Both the period and the fill factor influence the polarizer's extinction ratio; when the period increases, the latter decreases while increasing the fill factor results in higher extinction ratios at the expense of increased IL, as extensively investigated in our previous work [22]. The matching frequency is ruled by the polymer thickness and its refractive index and in the WGP it is found at a somewhat lower frequency than in the simple dielectric foil of equal thickness.

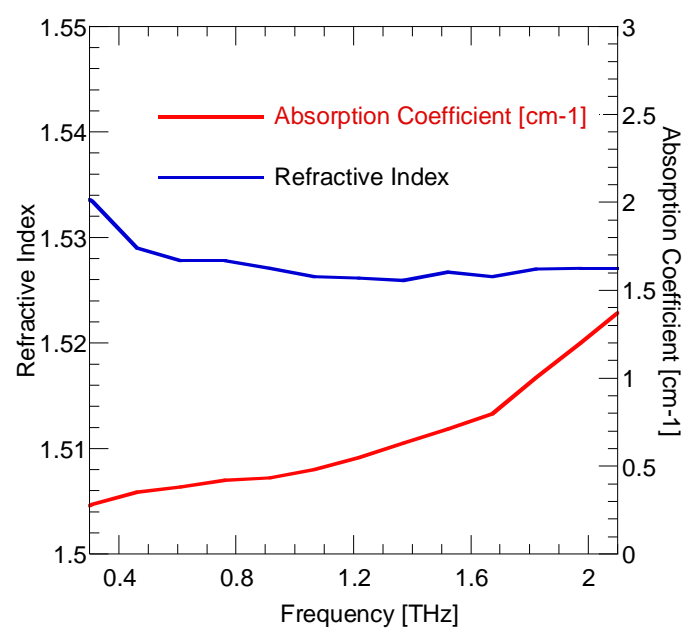

Fig. 1. Material properties of a 2-mm Zeonor sheet characterized by means of THz time-domain spectroscopy.

Before the fabrication process, a slab of $2 \mathrm{~mm}$ thickness of Zeonor was characterized using $\mathrm{THz}$ time domain spectroscopy in order to validate the properties reported in the literature at the $\mathrm{THz}$ frequency range, as shown in Fig. 1. The extracted refractive index is equal to 1.528 with minor dispersion in the spectral range under investigation while the absorption coefficient increases almost linearly from about 0 to $1.4 \mathrm{~cm}^{-1}$ in the 0.6 to $2.1 \mathrm{THz}$ range, corresponding to an almost constant imaginary part of the refractive index of approximately $10^{-3}$ and a loss tangent of approximately $1.3 \cdot 10^{-3}$. Given the very low absorption, is has not been possible to 
characterize the 40 and $100 \mu \mathrm{m}$ Zeonor foils individually because the sample measurements are comparable to the oscillation of the THz signal. However, a stack of $100 \mu \mathrm{m}$ thick foils was assembled and measured as well. The material parameters are fully consistent with the accuracy in estimating the foils and slab thicknesses and with the values found in the literature [24].

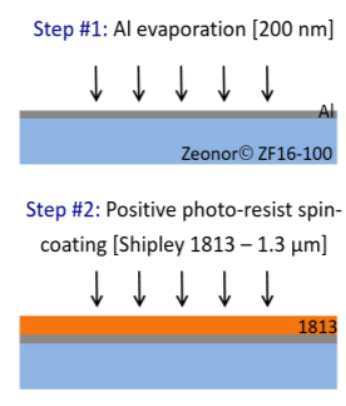

Step \#3: Photolithography (i-line UV)

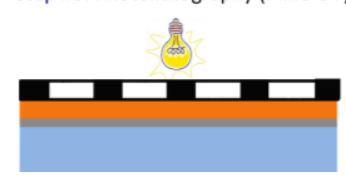

Step \#4: Development [MIF319] Pattern definition

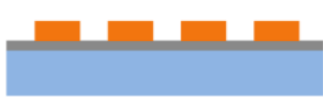

Step \#5: Al etching $\left[\mathrm{H}_{3} \mathrm{PO}_{4}: \mathrm{H}_{2} \mathrm{O}: \mathrm{CH}_{3} \mathrm{COOH}: \mathrm{HNO}_{3}\right]$

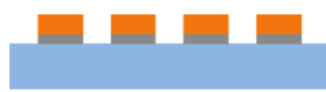

Step \#6: Residual photoresist removal $\left[\left(\mathrm{CH}_{3}\right)_{2} \mathrm{CO}\right]$

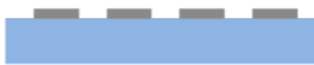

Fig. 2. Steps of the fabrication process of the investigated flexible THzpolarizers on Zeonor foils.

Two polarizers with a pitch $20 \mu \mathrm{m}$ and a fill factor of $70 \%$ $(\mathrm{w}=14 \mu \mathrm{m})$ were fabricated on low loss flexible and conformal 40 and 100 - $\mu$ m-thick Zeonor foils sized $2.54 \mathrm{~cm} \times 2.54 \mathrm{~cm}$, using standard optical photolithography techniques. With respect to our previous work [22] we doubled the pitch to facilitate the fabrication of the $\mathrm{THz}$ WGP on 40- $\mu$ m-thick Zeonor foils. The fabrication flow is described in Fig. 2. At first, 200nm of aluminium were thermally evaporated on Zeonor foils. Subsequently, a film of the positive photoresist S1813 from Shipley was deposited using spin coating at $3000 \mathrm{rpm}$ for 30 seconds, then backed at $115^{\circ} \mathrm{C}$ for 2 minutes. Its final thickness was $1.3 \pm 0.1 \mu \mathrm{m}$. The photolithography is carried out on the top surface using a Karl Suss MA150 mask aligner with a wavelength of $365 \mathrm{~nm}$ and intensity of $60 \mathrm{~mW} / \mathrm{cm}^{2}$. The sample were immersed in the developer MF319 for 50 seconds, rinsed with deionized water, dried with nitrogen, and, afterwards, baked at $120^{\circ} \mathrm{C}$ for 5 minutes. Finally, the exposed aluminium was wet-etched in $\mathrm{H}_{3} \mathrm{PO}_{4}: \mathrm{H}_{2} \mathrm{O}: \mathrm{CH}_{3} \mathrm{COOH}: \mathrm{HNO}_{3}=16: 2: 1: 1$. Then the samples were cleaned with acetone and isopropanol. Fig. 3 illustrates the WGP fabricated on $40 \mu \mathrm{m}$ Zeonor thickness fixed on a standard 1" rotation mount while the inset reports a microphotograph taken under an optical microscope in transmission mode with a diffraction limited $100 \times / 90$ microscope objective, where the black parts are the $\mathrm{Al}$ stripes, while the transparent - Zeonor.

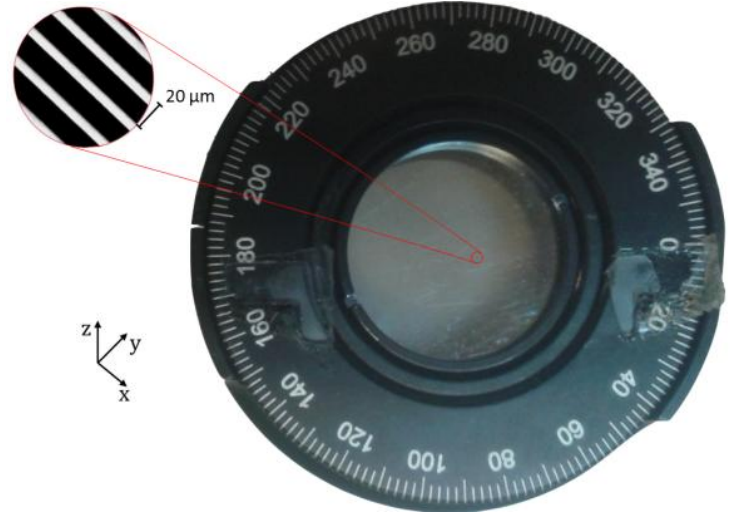

Fig. 3. Terahertz polarizer on a standard 1 inch mount. The inset reports a micrograph taken under the microspcope in transmission mode of a fabricated sample with $\mathrm{p}=20 \mu \mathrm{m}, \mathrm{w}=14 \mu \mathrm{m}$ on a Zeonor foil with $\mathrm{d}=40 \mu \mathrm{m}$.

The electromagnetic properties of the WPGs were investigated by means of $\mathrm{THz}$ time domain spectroscopy using a Menlo Systems TERA K15 all fiber-coupled spectrometer in transmission mode. The measurements were done in an atmosphere purged with nitrogen in order to prevent water absorption of $\mathrm{THz}$ radiation. A detailed description of the measurement process is reported in our previous work [22].

Fig. 4 illustrates the comparison of numerical (solid lines) and experimental (markers) results of the power ER for THz WGPs with $\mathrm{p}=20 \mu \mathrm{m}$ and $\mathrm{F}=70 \%$ on Zeonor foil with a thickness (d) of 40 and $100 \mu \mathrm{m}$. For both samples, the $\mathrm{THz}$ measurements agree very well with the simulations exhibiting an ER of $45 \mathrm{~dB}$ at $0.3 \mathrm{THz}$ which decreases as the frequency increases until the value of $30 \mathrm{~dB}$ at $2.1 \mathrm{THz}$. It is interesting to note that the slope of the ER depends on the polymer thickness, in particular for the WGP fabricated on $40 \mu \mathrm{m}$ Zeonor the slope is more regular with respect to the $100 \mu \mathrm{m}$ one due to the FabryPérot effects.

The influence of the polymer substrate on the polarizer performance is more evident in Fig. 5, which reports the comparison of experimental measurements (markers) and numerical (solid lines) results of the IL for the above samples. In fact, the WGPs exhibit two clearly different ILs that stay, however, between 0 and $1.1 \mathrm{~dB}$ for both samples. Interestingly, owing to the matching condition, the WGP on a $40 \mu \mathrm{m}$ substrate possesses an IL of $0.18 \mathrm{~dB}$ at around $2 \mathrm{THz}$, which is difficult to achieve since most common polymers used at $\mathrm{THz}$ exhibit great attenuation at $2 \mathrm{THz}$. Furthermore, the index matching condition is very important for use in combination with $\mathrm{THz}$ QCL sources because back-reflections are to be avoided [23].

Instead, the WGP on a $100 \mu \mathrm{m}$ foil shows the same trend observed in [22] where the IL approaches $0 \mathrm{~dB}$ at 0.9 and $1.7 \mathrm{THz}$. It is worth noting that the matching frequency of 
the two devices does not simply scale with the thickness of the polymer foil. Here, we stress the fact that the matching frequency is shifted to lower frequency for a larger fill factor and for a larger pitch. Finally, we envisaged also the possibility of engineering the polarizers on thinner foils for operation at higher frequency where THZ QCLs deliver larger power, but we are currently limited by the dynamic range of the available instrument in estimating large extinction ratios.

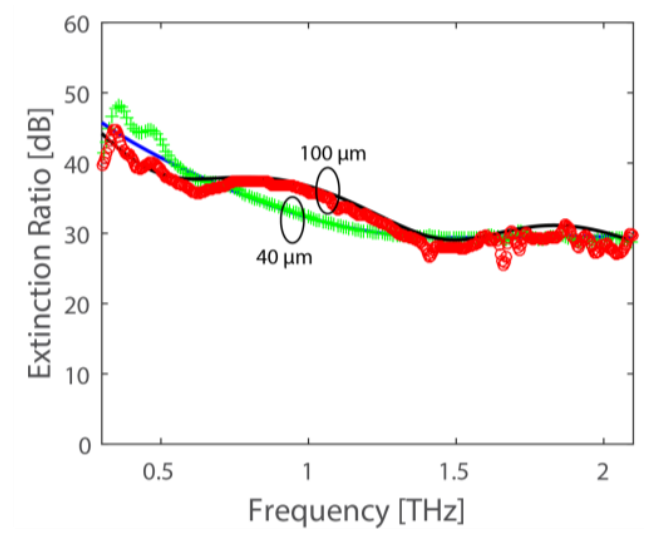

Fig. 4. Comparison of experimental measurements (markers) and numerical (solid lines) results of the extinction ratio for THz WGPs with $\mathrm{p}=20 \mu \mathrm{m}$ and $\mathrm{F}=70 \%$ on Zeonor foil 40 and $100 \mu \mathrm{m}$ thick.

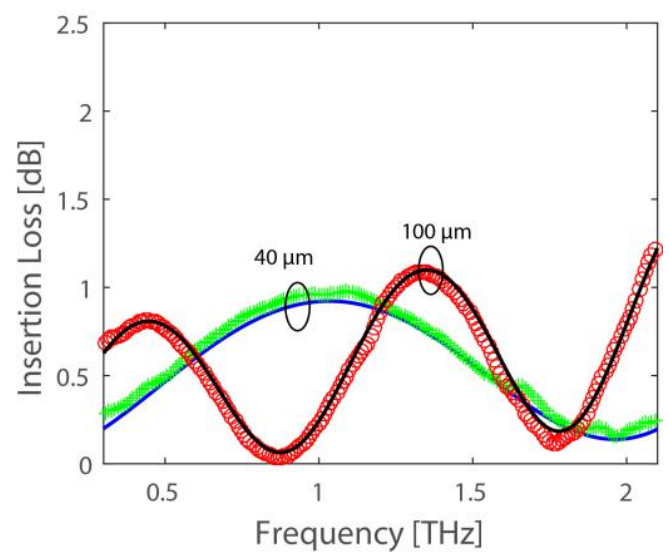

Fig. 5. Comparison of experimental measurements (markers) and numerical (solid lines) results of the insertion loss for THz WGPs with $\mathrm{p}=20 \mu \mathrm{m}$ and $\mathrm{F}=70 \%$ on Zeonor foil 40 and $100 \mu \mathrm{m}$ thick.

To sum up, in the present work we have demonstrated a rugged wire grid polarizer to be used with quantum cascade lasers. The investigated $\mathrm{THz}$ polarizers exhibit properties comparable to the commercial ones with ER and IL between 30 and $45 \mathrm{~dB}$ and 0 and $1.1 \mathrm{~dB}$ in the whole range under investigation. Moreover, the polarizers can be easily fabricated with low-cost techniques such as roll-to-roll and/or large-area electronics processes and promise to open the way for a new class of flexible and conformal $\mathrm{THz}$ devices.
This work was funded by the Italian Ministry of Foreign Affairs, Directorate General for the Country Promotion and by the Italian Ministry of Education, University and Research (Progetto Premiale THEIA). The authors would also like to acknowledge the contribution of the COST Action IC1208 (www.ic1208.com). They kindly thank Dr. Musci from ZEON Co. for the supply of materials.

\section{References}

[1] I. F. Akyildiz, J. M. Jornet, C. Han, Phys. Commun. 12, 16 (2014).

[2] M.C. Kemp, P.F. Taday, B.E. Cole, J.A. Cluff, A.J. Fitzgerald, W.R. Tribe, Proc. SPIE 5070, 44 (2003).

[3] M. Schirmer, M. Fujio, M. Minami, J. Miura, T. Araki, T. Yasui, Biomed. Opt. Express 1, 354 (2010).

[4] R.P. Cogdill, R.N. Forcht, Y. Shen, P.F. Taday, J.R. Creekmore, C.A .Anderson, J.K. Drennen, J. Pharm. Innov. 2, 29 (2007)

[5] Y.-C. Shen, Int. J. Pharm. 417, 48 (2011).

[6] A.G. Davies, A.D. Burnett, W. Fan, E.H. Linfield, J.E. Cunningham, Mater. Today 11, 18 (2008)

[7] J.F. Federici, B. Schulkin, F. Huang, D. Gary, R. Barat, F. Oliveira, D .Zimdars, Semicond. Sci. Technol. 20, S266 (2005).

[8] D. Saeedkia, Handbook of Terahertz Technology for Imaging, Sensing and Communications (Elsevier, 2013).

[9] N. Born, M. Reuter, M. Koch, M. Scheller, Opt. Lett. 38, 908 (2013).

[10] A. Ferraro, D.C. Zografopoulos, R. Caputo, R. Beccherelli, IEEE Photonics Technol. Lett. 28, 2459 (2016).

[11] A. Ferraro, D.C. Zografopoulos, R. Caputo, R. Beccherelli, IEEE J. Sel. Top. Quantum Electron. 2017, DOI 10.1109/JSTQE.2017.2665641.

[12] B. S.-Y. Ung, B. Weng, R. Shepherd, D. Abbott, C. Fumeaux, Opt. Mater. Express 3, 1242 (2013).

[13] J.-S. Li, D. Xu, J. Yao, Appl. Opt. 49, 4494 (2010).

[14] K. Altmann, M. Reuter, K. Garbat, M. Koch, R. Dabrowski, I. Dierking, Opt. Express 21, 12395 (2013).

[15] D.C. Zografopoulos, R. Beccherelli, Sci. Rep. 5, 13137 (2015).

[16] G. Isić, B. Vasić, D. C. Zografopoulos, R. Beccherelli, R. Gajić, Phys. Rev. Appl. 3, 064007 (2015).

[17] K. Iwaszczuk, A.C. Strikwerda, K. Fan, X. Zhang, R.D. Averitt, P.U. Jepsen, Opt. Express 20, 635 (2012).

[18] F. Yan, C. Yu, H. Park, E.P.J. Parrott, E. Pickwell-MacPherson, J. Infrared Millim. Terahertz Waves 34, 489 (2013).

[19] http://www.tydexoptics.com

[20] K. Imakita, T. Kamada, M. Fujii, K. Aoki, M. Mizuhata, S. Hayashi, Opt. Lett. 38, 5067 (2013).

[21] A. Isozaki, et al., Solid-State Sens. Actuators Microsyst. Transducers Eurosensors XXVII 2013 Transducers Eurosensors XXVII 17th Int Conf. On, IEEE, pp. 530-533 (2013).

[22] A. Ferraro, D. C. Zografopoulos, M. Missori, M. Peccianti, R. Caputo, R. Beccherelli, Opt. Lett. 41, 2009 (2016).

[23] M.S. Vitiello, G. Scalari, B. Williams, P.D. Natale, Opt. Express 23, 5167 (2015).

[24] A. Podzorov, G. Gallot, Appl. Opt. 47, 3254 (2008). 